\title{
The Hirsch spectrum: A novel tool for analyzing scientific journals
}

\author{
Fiorenzo Franceschini*, Domenico Maisano \\ Politecnico di Torino, Dipartimento di Sistemi di Produzione ed Economia dell'Azienda (DISPEA), Corso Duca degli Abruzzi 24, 10129 Torino, Italy
}

\section{A R T I C L E I N F O}

\section{Article history:}

Received 7 May 2009

Received in revised form 24 August 2009

Accepted 31 August 2009

\section{Keywords:}

Hirsch index

Hirsch spectrum

Journal's (co-)authors

Citations

Bibliometrics

Quality Engineering/Quality Management

journals

Journal qualimetrics

\begin{abstract}
A B S T R A C T
This paper introduces the Hirsch spectrum ( $h$-spectrum) for analyzing the academic reputation of a scientific journal. $h$-Spectrum is a novel tool based on the Hirsch $(h)$ index. It is easy to construct: considering a specific journal in a specific interval of time, $h$-spectrum is defined as the distribution representing the $h$-indexes associated to the authors of the journal articles. This tool allows defining a reference profile of the typical author of a journal, compare different journals within the same scientific field, and provide a rough indication of prestige/reputation of a journal in the scientific community. $h$-Spectrum can be associated to every journal. Ten specific journals in the Quality Engineering/Quality Management field are analyzed so as to preliminarily investigate the $h$-spectrum characteristics.
\end{abstract}

(c) 2009 Elsevier Ltd. All rights reserved.

\section{Introduction}

At the present time, there is a wide number of scientific journals with different status, prestige and diffusion, covering innumerable scientific disciplines. The most well-known tool to evaluate scientific journals is the ISI impact factor (ISI-IF), which was introduced by Garfield (1972). This indicator allows comparisons among different journals, provided that they belong to the same subject area (Amin \& Mabe, 2000). Although it shows some weak points, in many academic contexts it seems to be the main way for ranking journals (MacRoberts \& MacRoberts, 1987; Seglen, 1992; Seglen, 1997; Jennings, 1998; Glänzel \& Moed, 2002; Garfield, 2006; Brumback, 2008; Leydesdorff, 2009). Some main drawbacks of ISI-IF are: (i) not all scientific journals are indexed by Thomson Scientific, (ii) the limited time span (only citations accumulated within 2 years after the publication are considered) and (iii) the lack of coverage (citations in books, conference proceedings and dissertations are not included in the ISI list) (Seglen, 1997; Harzing, 2008; Thomson Reuters, 2009). Originally, ISI-IF was conceived to evaluate the diffusion of a journal but it had gradually become an indicator of prestige/reputation for the journal itself and, implicitly, for the authors of the papers there presented (Braun, Dióspatonyi, Zsindely, \& Zádor, 2007). In practice, the larger ISI-IF, the more prestigious the journal.

For a potential author, the scientific reputation of the past and current authors of one journal is a reason of attraction. Reputation/prestige of the journal editor-in-chief and editorial board members, and presence of papers submitted by eminent scientists are some other possible reasons for preferring one journal to another. However, these evaluations are often subjective and not very reliable. Braun, Glänzel, \& Schubert (2006) proposed using the Hirsch $(h)$ index for evaluating and comparing scientific journals. Specifically, $h$ is defined as the number such that, for a general group of papers, $h$ papers received at least $h$ citations while the other papers received no more than $h$ citations (Hirsch, 2005, 2007). $h$ was originally introduced by Hirsch

\footnotetext{
* Corresponding author. Tel.: +39011 5647225; fax: +39 0115647299.

E-mail address: fiorenzo.franceschini@polito.it (F. Franceschini).
} 
in order to evaluate the quantity and the diffusion of one researcher's scientific production. Ever since its introduction, $h$ received much attention. This indicator has many merits (i.e. it is synthetic, robust, simple to calculate and with immediate intuitive meaning) and some weak points; both have been abundantly pointed out in the literature (Moed, 2005; Egghe, 2006; Glänzel, 2006; Kelly \& Jennions, 2006; Rousseau, 2006; Saad, 2006; Bornmann \& Daniel, 2007; Costas \& Bordons, 2007; Orbay, Karamustafaoglu, \& Oner, 2007; Schreiber, 2007; Van Raan, 2006; Wendl, 2007; Harzing \& van der Wal, 2008; Mingers, 2009; Franceschini and Maisano, 2009a). Another tangible sign of the popularity of $h$ is the appearance of many proposals for new variants and improvements, including the above-mentioned $h$-index for journals (Lehmann, Jackson, \& Lautrup, 2005; Banks, 2006; Batista, Campiteli, Kinouchi, \& Martinez, 2006; Braun et al., 2006; Lehmann, Jackson, \& Lautrup, 2006; BiHui et al., 2007; Burrell, 2007a,b; Castillo, Donato, \& Gionis, 2007; Katsaros, Sidiropoulos, \& Manolopous, 2007; Sidiropoulos, Katsaros, \& Manolopoulos, 2007; Schreiber, 2008; Antonakis \& Lalive, 2008; Woeginger, 2008; Franceschini and Maisano, 2009b).

Coming back to the $h$-index for journals, it is calculated taking into consideration the articles published by a specific journal in a precise time period (e.g. 1 year). Unfortunately, this indicator has a significant limitation. Considering a generic journal, the citation accumulation process of the papers requires a certain amount of time to become stable-according to some authors, this period is about 5 years in the engineering field (Amin \& Mabe, 2000; Castillo et al., 2007; Harzing, 2008). Thus, $h$ for journals is not suitable to evaluate the most recently published journals and, much less, to compare them with other past journals. Besides, being sensitive to the number of papers per issue, this indicator - if calculated on a yearly basis - tends to favour journals with many papers/issues per year. In fact, a high number of articles per year are not necessarily an element in favour of a journal with respect to another.

The goal of this paper is to introduce the Hirsch spectrum ( $h$-spectrum), a new tool that is derived from $h$ and defined as the distribution representing the $h$-indexes associated to the authors of a specific journal, in a specific interval of time. The term "spectrum" is originated from the fact that this distribution provides an image of the author population of one journal for a period of interest. In our view, $h$-spectrum represents a different way for evaluating and comparing the reputation of journals (indexed by Thomson Scientific or not).

More in detail, $h$-spectrum can be used for several practical purposes, respectively:

- to make a comparison among journals within the same scientific field;

- to define the profile of the "typical authors" of a specific journal. This profile may represent a reference for other (potential) authors;

- extending the idea of the previous point, to define a reference of the "typical researcher" of a specific discipline (both in terms of productivity and diffusion, which are the basic reasons why we decided to use $h$ );

- to help a journal's editorial board to periodically monitor the effect of the paper selection policy, from the point of view of the population of the journal authors. In this sense, $h$-spectrum may become an indirect indicator of editorial strategy;

- to provide a rough indication on the prestige/influence of a journal on the scientific community.

To focalize our preliminary analysis, the $h$-spectrum study is circumscribed to a particular discipline. We analyzed some journals in the Quality Engineering and Quality Management area.

The remaining of this paper is organised into three sections. Section 2 illustrates the methodology used in the analysis and shows some preliminary results. Section 3 focuses on some peculiar aspects of the $h$-spectrum and makes a brief comparison with ISI-IF. Section 4 identifies several ideas for further research activities, which may originate from this work. Finally, the conclusions are given, summarising the original contribution of this paper.

\section{Methodology and preliminary results}

The $h$-spectrum analysis can be divided in two distinct activities:

1. construction and comparison of the $h$-spectra related to different journals in the same reference year, so as to investigate how the $h$-spectrum changes from journal to journal;

2. construction and comparison of the $h$-spectra related to the same journal(s) in different periods of time, so as to investigate how a journal's $h$-spectrum tends to change over time.

\subsection{Comparison among different journals in the same year}

The purpose of this activity is to make a comparison among journals published in the same year (for example in 2008). We selected 10 different Quality journals, from the most popular and representative in this discipline (ASQ, 2009; Harzing, 2009; Thomson Reuters, 2009). These journals have different publishers and only a small portion of them (see Table 1) is indexed by Thomson Scientific. Table 1 also reports the journals' acronyms.

For each journal, all the (co-)authors of a paper published in the reference year (i.e. 2008) are identified. Then, the corresponding $h$-index of each (co-)author is calculated. Finally, the distribution of the (co-)authors' $h$-indexes is constructed according to the following assumptions/conventions: 
Table 1

List of the 10 journals selected among the Quality Engineering/Quality Management area.

\begin{tabular}{|c|c|c|c|c|}
\hline Journal name & Acronym & Publisher & $\begin{array}{l}\text { Indexed by Thomson } \\
\text { Scientific }\end{array}$ & $\begin{array}{l}\text { Year(s) for which } \\
h \text {-spectrum is calculated }\end{array}$ \\
\hline Journal of Quality Technology & JQT & ASQ & Yes & 2004-2008 \\
\hline Quality Engineering & $\mathrm{QE}$ & ASQ & No & 2004-2008 \\
\hline Quality and Quantity & QQ & Springer & Yes & 2008 \\
\hline Quality and Reliability Engineering International & QREI & Wiley & Yes & 2008 \\
\hline Quality Management Journal & QMJ & ASQ & No & 2008 \\
\hline Managing Service Quality & MSQ & Emerald & No & 2008 \\
\hline Journal of Quality in Maintenance Engineering & JQME & Emerald & No & 2008 \\
\hline International Journal of Quality and Reliability Management & IJQRM & Emerald & No & 2008 \\
\hline Quality Progress & $\mathrm{QP}$ & ASQ & No & 2004-2008 \\
\hline
\end{tabular}

- All the different (co-)authors of one journal have the same importance. Thus, their $h$-indexes are not weighted in inverse proportion to the number of (co-)authors of the corresponding paper(s).

- The $h$-index of a (co-)author, who publishes more than one article in a journal during the period of interest, is counted (only) once.

- For simplicity, the $h$-index of each author is calculated taking into account the scientific publications and citations accumulated up to the moment of the analysis (in our case, May 2009). In fact, it should be remarked that the $h$-index of a scientist tends to increase over time, because of the gradual accumulation of publications/citations. With a bit more effort-the analysis can be developed considering the publications/citations accumulated up to the journal publication date, excluding the subsequent ones. However, since the average time growth rate of $h$-index is relatively small (in engineering disciplines especially), the real variations in the $h$-spectra would be reasonably limited (Burrell, 2007b). To avoid any misunderstanding, when calculating a journal's $h$-spectrum two parameters have to be stated: (i) the period of interest in which the journal authors are identified (e.g. the whole year 2008) and (ii) the precise moment in which author $h$-indexes are calculated (in our case, May 2009).

The output of the first analysis activity is illustrated in Fig. 1, showing the $h$-spectra related to the 10 Quality Engineering/Quality Management journals reported in Table 1.

At a first glance, all these distributions are right-skewed and have a characteristic profile, which is approximately decreasing. Analyzing the distributions in more detail, some interesting aspects emerge. Fig. 2 shows the $h$-index average value $(\bar{h})$, the corresponding standard deviation $(s)$ and the number of authors $(N)$ related to each journal. Journals are sorted in descending order with respect to $\bar{h}$. It can be noticed that, despite their similar shape, distributions are appreciably different in terms of values of $\bar{h}$ and $s$.

Furthermore, it is interesting to notice that - considering the same journal $-\bar{h}$ and s have generally similar values. Their empirical correlation is nearly linear with a relatively high coefficient of determination $\left(R^{2} \approx 0.87\right.$, see Fig. 3$)$. On the other hand, there is no correlation between $\bar{h}$ and $N$ or $s$ and $N\left(R^{2} \approx 0\right)$.

On the basis of this result, it seems quite appropriate using $\bar{h}$ as a synthetic indicator to perform quick evaluations and comparisons among different $h$-spectra. Nevertheless, we want to emphasise the fact that the $h$-spectrum is more than a simple numerical indicator (like the ISI-IF or the $h$-index for journals); it is a distribution (Franceschini, Galetto, \& Maisano, 2007; Franceschini, Galetto, Maisano, \& Mastrogiacomo, 2008; Chapman, 2009).

\subsection{Time evolution of the h-spectrum}

This analysis is aimed at finding how the $h$-spectrum changes over time. Three of the previous 10 journals are selected - i.e. JQT, QE and QP - extending the $h$-spectrum analysis to a period of five consecutive years (from 2004 to 2008 ). Fig. 4 reports the resulting $h$-spectra.

For each journal, the $h$-spectrum seems relatively robust and stable over the five examined years (see Fig. 5). Two possible reasons of this relative stability could be:

- authors of a particular journal tend to be "attracted" to it over the years;

- the editorial board policy tends to be consistent over time.

Since, there can be small variations from 1 year to the next, we noticed that the characteristic shape of a journal's $h$ spectrum becomes more and more consolidated by increasing the reference time period. This aspect is shown in Fig. 6 , which reports the $h$-spectra of three journals (i.e. JQT, QE and QP), constructed considering three different periods of interest (1 year, 3 years and 5 years, respectively). It can be seen that the difference among the journal $\bar{h}$ values becomes clearer when the reference time period increases. In this sense, $h$-spectrum can be used as an indicator of one journal's prestige. 

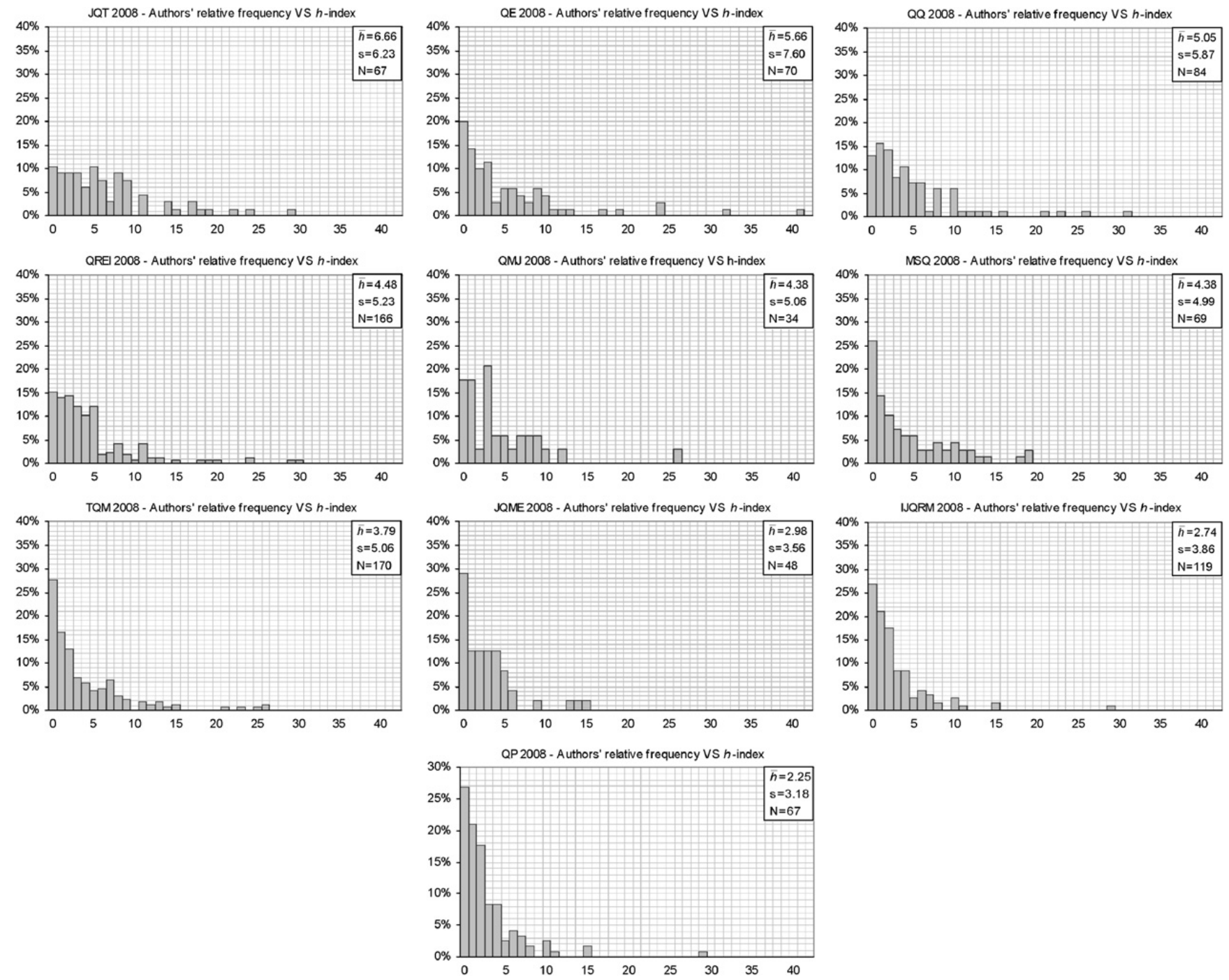

Fig. 1. $h$-Spectra (authors' relative frequency VS $h$-index) for 10 Quality Engineering/Quality Management journals, in the year 2008 . Journal acronyms are indicated in Table 1 . For each journal, the authors' $h$-index average value $(\bar{h})$, the corresponding standard deviation $(s)$ and the number of authors $(N)$ are reported. Date of the analysis: May 2009.

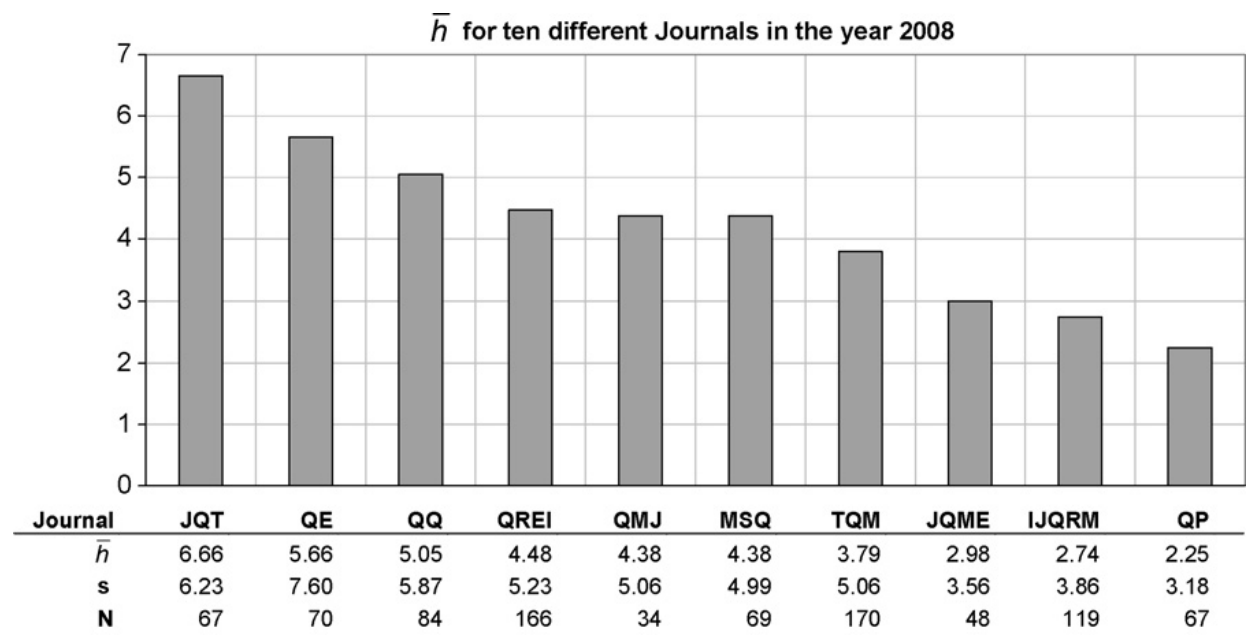

Fig. 2. Synthetic results of the analysis of 10 Quality Engineering/Quality Management journals, in the year 2008. The table reports the authors' $h$-index average value $(\bar{h})$, the corresponding standard deviation $(s)$ and the number of authors $(N)$ related to each journal. In the graph, journals are sorted in descending order with respect to $\bar{h}$. Date of the analysis: May 2009. 


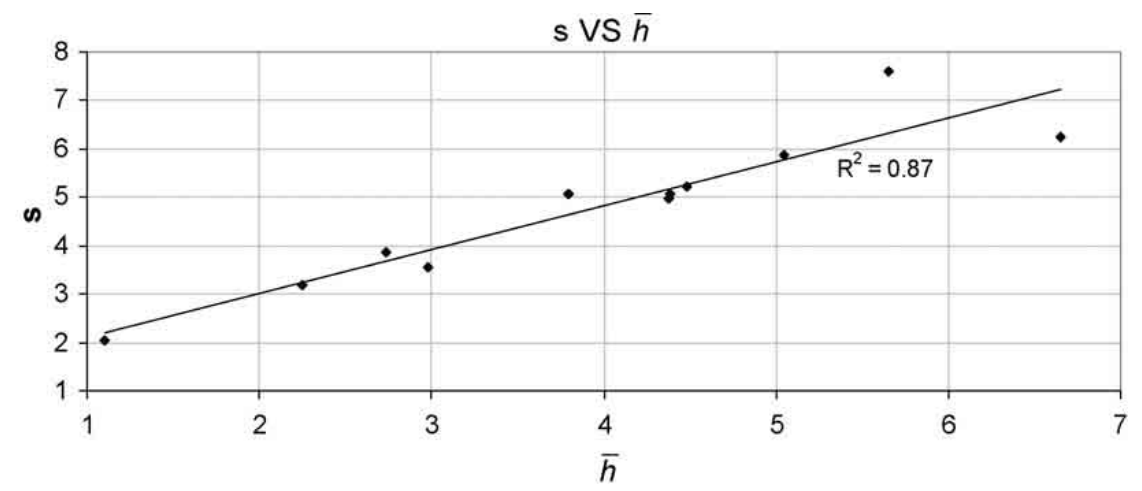

Fig. 3. Relationship between s and $\bar{h}$ related to the $h$-spectra in Fig. 1, for 10 Quality Engineering/Quality Management journals.

\subsection{Data source}

Citation statistics are collected using Google Scholar (GS) as a search engine. It was decided to use this database (i) because of its grater coverage and (ii) because it can be easily accessed through the Publish or Perish (PoP) freeware software, specially designed for citation analysis with GS (Meho \& Yang, 2007; Harzing, 2008; Harzing and van der Wal, 2008). Nevertheless, the analysis can be repeated using other databases, like Web of Science or Scopus.

One of the major problems encountered in our analysis is represented by homonym authors. In general, authors with common names or authors identified only by the surname and the first name initials - rather than full first name(s) - are subject to this kind of problem. The practical effect is that contributions of different homonym authors are erroneously added up, with the result of "inflating" one author's $h$. Luckily, these "suspected" authors can be detected and then excluded from the analysis quite easily. However, since they represent just a small part of the available information, the loss of information is not significantly relevant for our purpose.

\section{Further considerations on the $h$-spectrum}

\subsection{Author's reputation}

We think that $h$-spectrum can be a reliable tool for evaluating a journal at the very moment of the publication, despite the fact that it is based on the publications/citations accumulated before the publication of the examined journal. There are empirical proofs of the fact that citations that a new paper will receive in the future are generally consistent with the citations accumulated by previous papers of the same author, that is to say the author's reputation (Castillo et al., 2007). Being the number of authors per journal quite large (typically more than fifty authors per year, as shown in Fig. 2), it is reasonable to assume that the authors' reputation will be generally respected.

\subsection{Information content of h-spectrum}

$h$-Spectrum represents a "snapshot" of the author population of a specific journal and can be a reference for researchers within the area of interest (Quality Engineering/Quality Management in this case). Assuming that an academic researcher with $h=3$ compares himself with the authors of a journal in 2008, what is the result? Using the $h$-spectra shown in Fig. 1, he will fall on the 32nd percentile of the JQT $h$-spectrum, the 50th percentile of the QE $h$-spectrum, the 37th percentile of the QQ $h$-spectrum and so on. Thus, $h$-spectrum can be interpreted as a kind of "identity card" for scientific journals, since it provides indications about the authors who populate it.

Examining the $h$-spectrum, we can observe some practical effects of the strategic choices of one journal's editorial board. For instance, in spite of being a very prestigious and popular journal in the Quality area, QP has a more left-adjusted $h$ spectrum than the other journals. The reason is that it is open to eminent professionals and industrial managers, with enviable professional careers in the industry, but with relatively low $h$-indexes.

\section{3. $h$-Spectrum as a complement to ISI-IF}

It is worthwhile remarking the difference between $h$-spectrum, which is related to the reputation of one journal's authors, and ISI-IF (or other traditional bibliometric indicators), which is related to the citations effectively accumulated by one journal's articles. Generally speaking, the academic reputation of a journal's author group is not the equivalent of the reputation of the journal, as well as not the equivalent of the influence of the journal. For this reason, these different indicators represent two complementary ways to evaluate/compare scientific journals. For example, a combined use of $h$-spectrum and ISI-IF can be performed for identifying the following situations: 

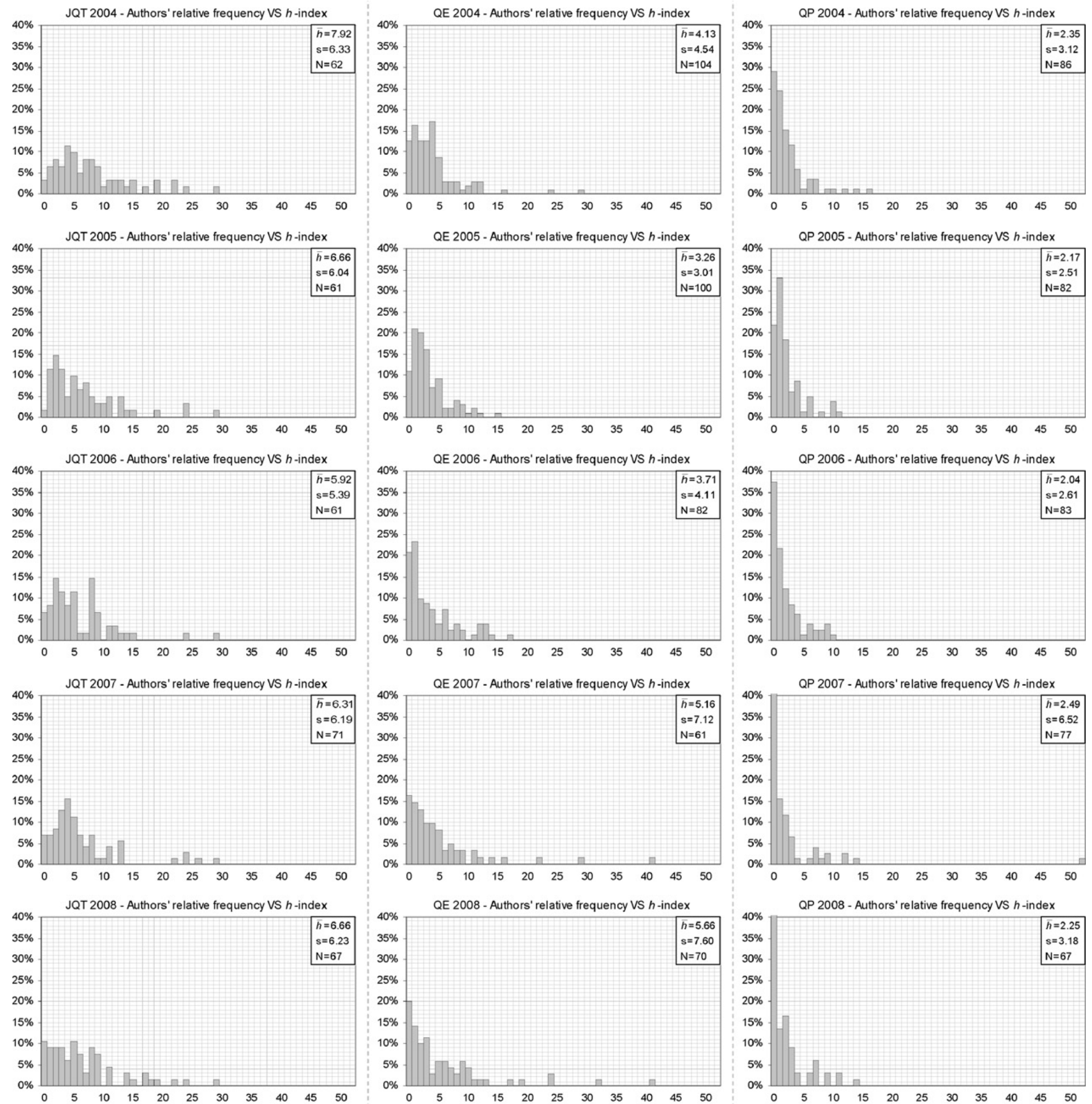

Fig. 4. $h$-Spectra for three Quality Engineering/Quality Management journals (JQT, QE and QP), in five consecutive years (from 2004 to 2008 ). For each journal, the authors' $h$-index average value $(\bar{h})$, the corresponding standard deviation $(s)$ and the number of authors $(N)$ are reported. Date of the analysis: May 2009.

1. Journals with high $\bar{h}$-spectrum values but few received citations. This can be the case of relatively recent journals which are still struggling to become popular in the scientific community.

2. Journals containing articles with a high number of citations, submitted by (co-)authors with low $h$-indexes. This can be the case of journals open beyond the academic world, for instance to professionals and industrial managers (like QP, as mentioned before). Alternatively, they can be journals with a relatively large group of young (co-)authors, consisting of brilliant young researchers with relatively low citation indexes.

Furthermore, the common points of critique on the ISI-IF do not necessarily hold for $h$-spectrum, due to the different nature of these tools. Here is presented a brief description of the major ones. 


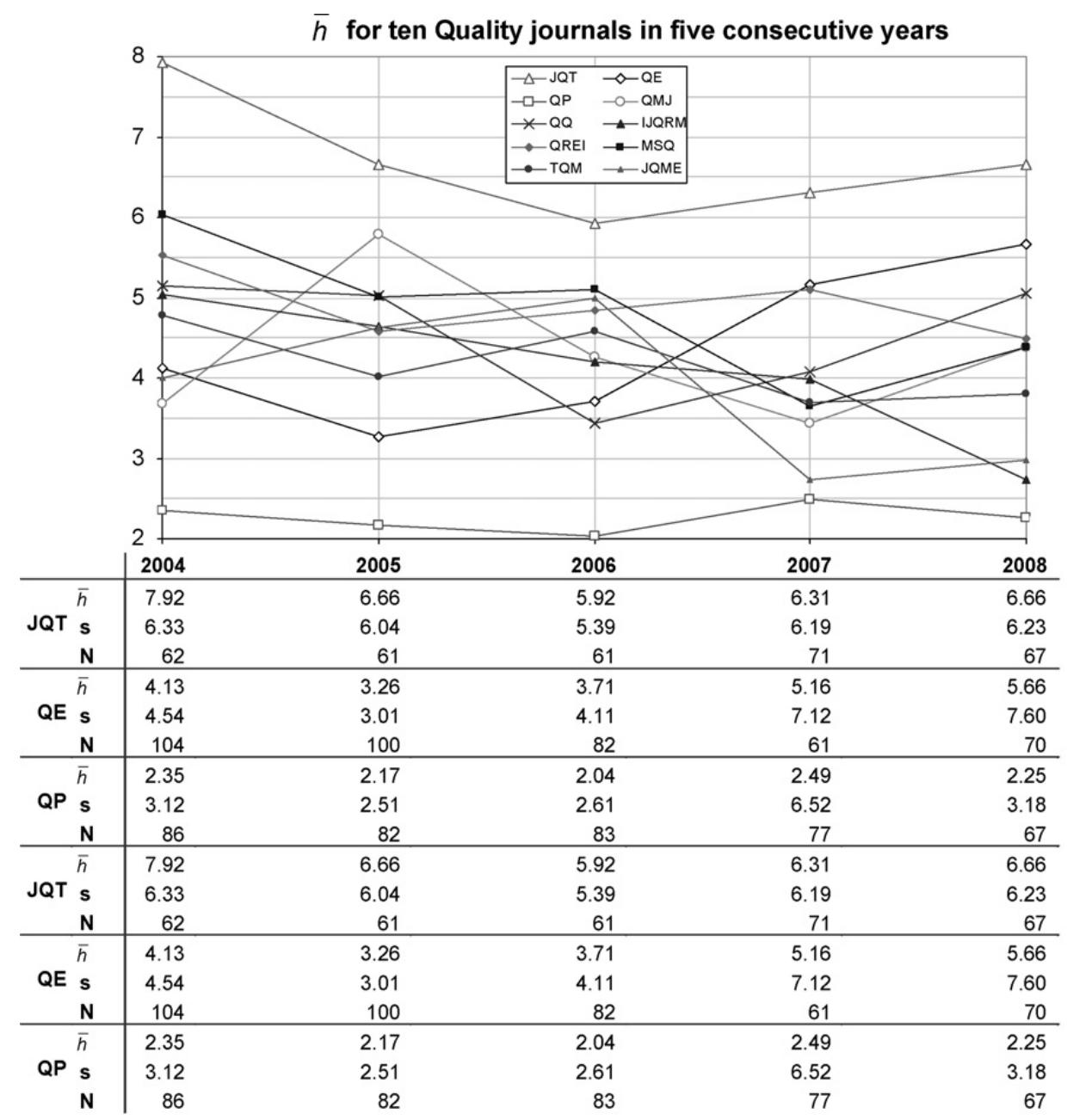

Fig. 5. Time evolution of the $h$-index average value $(\bar{h})$, the corresponding standard deviation $(s)$ and the number of authors $(N)$ for three journals (JQT, QE and QP) in five consecutive years (from 2004 to 2008).

- As well as ISI-IF, $h$-spectrum should not be used for comparing journals of different disciplines owing to the different citation rates (Amin \& Mabe, 2000; Antonakis \& Lalive, 2008).

- Differently from ISI-IF, $h$-spectrum can cover every journal (not only those indexed by Thomson Scientific).

- $h$-Spectrum can be calculated at the very moment of the journal publication, while ISI-IF cannot be calculated sooner than 1-2 years after the publication.

- As already highlighted, $h$-spectrum is more than a simple numerical indicator (like the ISI-IF or the $h$-index for journals). It is a distribution.

- The ISI-IF's variability is related to the size of the journal, in terms of articles published per annum. Small titles (less than 35 papers per annum) on average vary in ISI-IF by more than $+/-40 \%$ from 1 year to the next, while larger titles (more than 150 articles per annum) have a smaller fluctuation of $+/-15 \%$ (Amin \& Mabe, 2000). On the other hand, $h$-spectrum's variability does not seem to be influenced by the size of the journal. Fig. 3 shows that the standard deviation ( $s$ ) roughly depends on $\bar{h}$, which seems to be independent on the number of articles.

If on the one hand, $h$-spectrum does not suffer from many of the previous criticisms, on the other hand, it has the potential problem that - being based on $h$-index - it could be subjected to the criticisms made to $h$-index itself. Here follows a list of the most typical points. Many of them are generally true for any indicator in citation analysis: (i) $h$ does not take into account multiple co-authorship (Burrell, 2007a; Schreiber, 2008); (ii) $h$ does not take into account self-citations (Schreiber, 2007; Burrell, 2007a); (iii) $h$ is not useful for cross-disciplinary comparisons because citation rates and scholarly productivity vary considerably among disciplines (e.g. physics, medicine, engineering) (Antonakis \& Lalive, 2008; Batista et al., 2006; Braun et al., 2006); (iv) $h$ does not take into account the age of publications (Sidiropoulos et al., 2007). In theory, it would make sense giving more weight to most recent articles of a scientist and less weight to the eldest (Sidiropoulos et al., 2007); (v) $h$ is unfavourable to young brilliant scientists with few highly diffused articles (Sidiropoulos et al., 2007); (vi) $h$ does not consider the publication type. For example review articles, open access articles, papers addressing "hot topics" or in fields shared by large communities will often receive far more citations than other papers, all other things being equal (Castillo et al., 2007); (vii) the $h$-index for a scientist can be easily calculated by using public databases like WoS or GS (Meho \& Yang, 2007). 

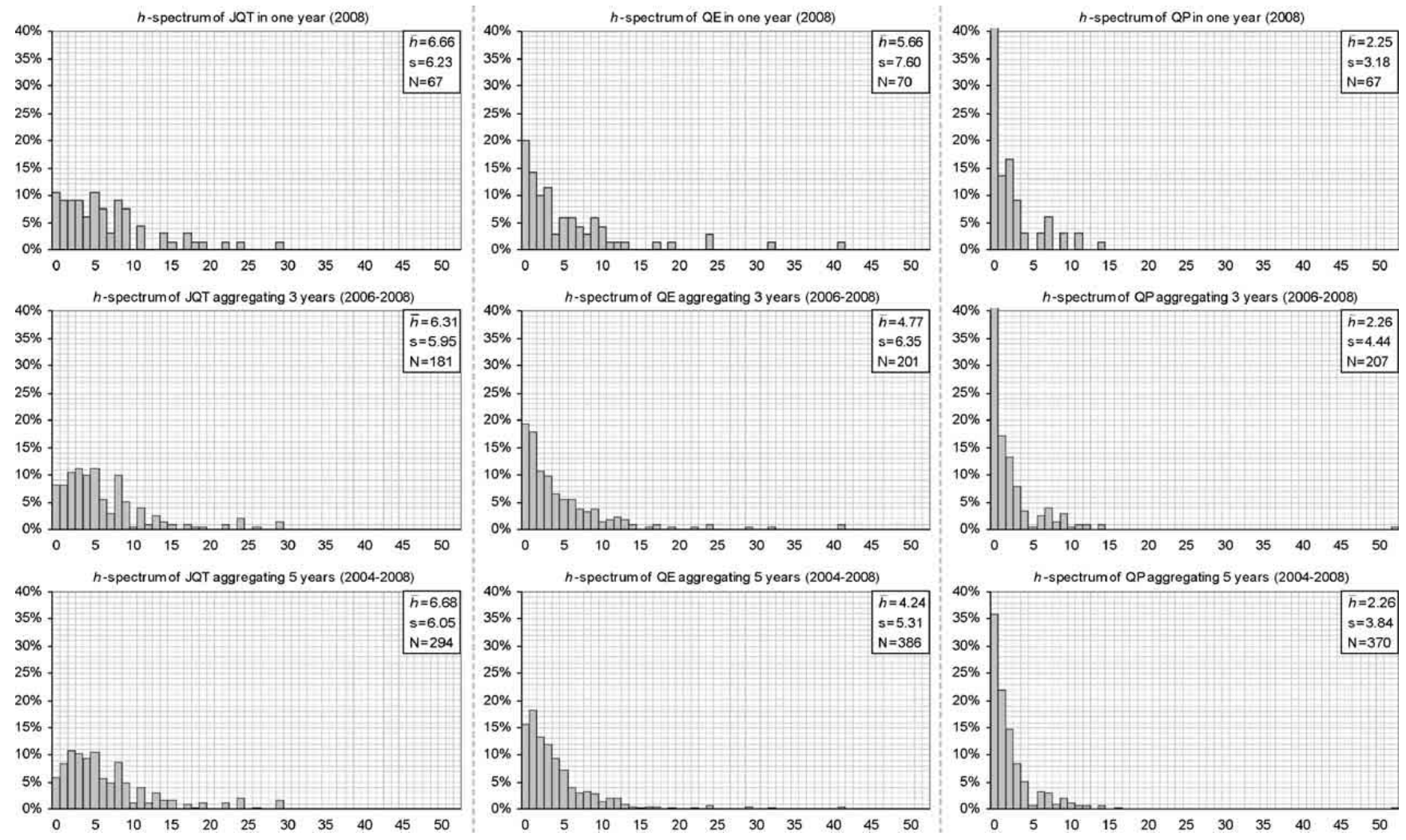

Fig. 6. $h$-spectra for three Quality Engineering/Quality Management journals (JQT, QE and QP), calculated considering three different reference time periods (respectively, 1 year, 3 years and 5 years). For each journal, the authors' $h$-index average value $(\bar{h})$, the corresponding standard deviation $(s)$ and the number of authors $(N)$ are reported. It can be seen that the larger the time period, the more consolidated the journal's $h$-spectrum. Date of the analysis: May 2009.

Unfortunately, their information can be affected by citation errors-for instance caused by homonymous author names, typographical errors in the source papers, or errors due to some nonstandard reference formats (Bornmann \& Daniel, 2007; Harzing \& van der Wal, 2008).

Being $h$-spectrum constructed considering a rather large number of (co-)authors (generally larger than 60 , as seen in Fig. 5), most of the possible "distortions" - consequent upon the use of $h$ - reasonably compensate each other. In other words, the effect of self-citation, multiple authorship, different age of publications, different age of authors and database errors should reasonably be spread over the (co-)authors, and there is no realistic reason why the $h$-spectrum of one journal should be more biased than that of another journal. In our opinion, the extent of these problems become more important when evaluating and comparing a small number of scientists (e.g. 5-10), using $h$ alone.

In spite of this, due to its characteristics of robustness, easy calculation, immediate intuitive meaning and synthesis, $h$ is the most suitable indicator for the construction of a journal-spectrum. The fact remains that it could be constructed on the basis of other indicators, which are not affected by (some) of the previous problems. For example, a spectrum could be based on the $h$-rate or AR-index-two $h$-based indicators, which do not favour scientists with long careers (Burrell, 2007b; BiHui, LiMing, Rousseau, \& Egghe, 2007).

\section{Open issues}

Several ideas for further research activities may originate from this work. Here follows a list of the most interesting ones:

- Repeating the analysis using other databases (i.e. Web of Science, Scopus or the DBLP digital library), so as to investigate possible differences in the results.

- Introducing a weighting system for author contribution, which takes account of multiple authorships and (co-)authors with multiple papers in the same period of interest, when determining the $h$-spectrum of a journal.

- Building a software application so as to automatically query GS and construct the $h$-spectrum for a specific journal and a specific year. This automatic procedure should include a proper filter to identify and remove "suspected" authors, with erroneous or nonsensical $h$-values.

- Building a mathematical model representing the $h$-spectrum.

- Extending the use of $h$-spectrum beyond scientific journals, so as to evaluate and compare academic research groups, university departments or - more in general - organizations made up of scientists, on the basis of their scientific reputation (Chapman, 2009). 
- Establishing which type of $h$-spectrum is better or which is the better mix between old scientists' or young scientists' $h$-values.

\section{Conclusions}

The main novelty of this paper is the introduction and discussion of the $h$-spectrum, a new tool - based on the $h$-index - that can be used for three major purposes: (i) providing a reference for the (potential) authors of a scientific journal; (ii) performing rough comparisons between different journals within the same scientific field (journal academic reputation); (iii) helping a journal's editorial staff to periodically monitor the effect of the paper selecting policy.

The results of a preliminary analysis, which is carried out considering 10 journals in the Quality Engineering and Quality Management field, are shown. It is interesting to observe that the $h$-spectrum has a peculiar shape and it is rather robust over the years. The $h$-spectrum can be calculated for each journal (not necessarily those indexed by Thomson Scientific) at the very moment of the journal publication-differently from the ISI-IF, that is calculated 1-2 years after the publication.

Several ideas for further research activities may originate from this work. In particular, it would be interesting to extend the analysis to a wider set of journals, considering a wider time horizon, and to build a mathematical model representing the $h$-spectrum.

\section{References}

American Society for Quality, (2009) http://www.asq.org/.

Amin, M., \& Mabe, M. (2000). Impact Factors: Use and Abuse. Elsevier Science. Perspectives in Publishing, no. 1, October 2000. http://www.elsevier.com Antonakis, J., \& Lalive, R. (2008). Quantifying scholarly impact: IQp versus the Hirsch h. Journal of the American Society for Information Science and Technology, 59(6), 956-969.

Banks, M. G. (2006). An extension of the Hirsch index: indexing scientific topics and compounds. Scientometrics, 69(1), 161-168.

Batista, P. D., Campiteli, M. G., Kinouchi, O., \& Martinez, A. S. (2006). Is it possible to compare researchers with different scientific interests? Scientometrics, $68(1), 179-189$

BiHui, J., LiMing, L., Rousseau, R., \& Egghe, L. (2007). The R- and AR-indices: complementing the h-index. Chinese Science Bulletin, $52(6), 855-963$.

Bornmann, L., \& Daniel, H. D. (2007). What do we know about the h index? Journal of the American Society for Information Science and Technology, 58(9), 1381-1385.

Braun, T., Glänzel, W., \& Schubert, A. (2006). A Hirsch-type index for journals. The Scientist, 69(1), 169-173.

Braun, T., Dióspatonyi, I., Zsindely, S., \& Zádor, E. (2007). Gatekeeper index versus impact factor of science journals. Scientometrics, 71(3), $541-543$.

Brumback, R. (2008). Worshipping false idols: the impact factor dilemma. Journal of Child Neurology, 23(4), 365-367.

Burrell, Q. L. (2007a). On the h-index, the size of the Hirsch core and Jin's A-index. Journal of Informetrics, 1(2), 170-177.

Burrell, Q. L. (2007b). Hirsch index or Hirsch rate? Some thoughts arising from Liang's data. Scientometrics, 73(1), 19-28.

Castillo, C., Donato, D., \& Gionis, A. (2007). Estimating number of citations using author reputation. In String Processing and Information Retrieval. Heidelberg: Springer Berlin., p. 107-117

Chapman, S. (2009). The H index distribution in the School of Public Health at the University of Sydney, November 2008. http://www.health.usyd.edu.au/ research/H-index-public.pdf

Costas, R., \& Bordons, M. (2007). The h-index: advantages, limitations and its relation with other bibliometric indicators at the micro level. Journal of Informetrics, 1(3), 193-203.

Egghe, L. (2006). Theory and practise of the g-index. Scientometrics, 69(1), 131-152.

Franceschini, F., Galetto, M., \& Maisano, D. (2007). Management by Measurement: Designing Key Indicators and Performance Measurement Systems. Berlin: Springer Verlag.

Franceschini, F., Galetto, M., Maisano, D., \& Mastrogiacomo, L. (2008). Properties of performance indicators in operations management: a reference framework. The International Journal of Productivity and Performance Management, 57, 137-155.

Franceschini, F., \& Maisano, D. (2009a). Analysis of the Hirsch index's operational properties. European Journal of Operational Research.

Franceschini, F., \& Maisano, D. (2009b). The Hirsch index in manufacturing and Quality engineering. Quality and Reliability Engineering International, doi:10.1002/qre.1016

Garfield, E. (1972). Citation analysis as a tool in journal evaluation. Science, 178(60), 471-479.

Garfield, E. (2006). The history and meaning of the journal impact factor. JAMA, 295(1), 90-93.

Glänzel, W., \& Moed, H. F. (2002). Journal impact measures in bibliometric research. Scientometrics, 53(2), 171-193.

Glänzel, W. (2006). On the opportunities and limitations of the h-index. Science Focus, 1(1), 10-11.

Harzing, A. W. (2008). Reflections on the h-index. http://www.harzing.com/

Harzing, A. W., \& van der Wal, R. (2008). Google Scholar as a new source for citation analysis. Ethics in Science and Environmental Politics, 8(11), 61-73.

Harzing, A.W. (2009) http://www.harzing.com/jql.htm.

Hirsch, J. E. (2005). An index to quantify an individual's scientific research output. In Proceedings of the National Academy of Sciences of the United States of America, vol. 102 (pp. 16569-16572).

Hirsch, J. E. (2007). Does the h index have predictive power? In Proceedings of the National Academy of Sciences of the United States of America, vol. 104, n. 49 p. 19193-19198

Katsaros, D., Sidiropoulos, A., \& Manolopous, Y. (2007 April 27). Age Decaying H-Index for Social Network of Citations. In Proceedings of Workshop on Social Aspects of the Web Poznan, Poland (p. 2007).

Kelly, C. D., \& Jennions, M. D. (2006). The h index and career assessment by numbers. Trends in Ecology E Evolution, 21(4), 167-170.

Jennings, C. (1998). Citation data: the wrong impact? Nature Neuroscience, 1, 641-642.

Lehmann, S., Jackson, A. D., \& Lautrup, B. E. (2005). Measures and Mismeasures of Scientific Quality. http://arxiv.org/abs/physics/0512238

Lehmann, S., Jackson, A. D., \& Lautrup, B. E. (2006). Measures for Measures. Nature, 444, 1003-1004.

Leydesdorff, L. (2009). How are new citation-based journal indicators adding to the bibliometric toolbox? to appear on Journal of the American Society for Information Science \&' Technology.

MacRoberts, M., \& MacRoberts, B. (1987). Problems of citation analysis: a critical review. Journal of the American Society for Information Science and Technology, 40(5), 342-349.

Meho, L., \& Yang, K. (2007). Impact of data sources on citation counts and ranking of LIS faculty: Web of Science versus Scopus and Google Scholar. Journal of the American Society for Information Science and Technology, 58(13), 2105-2125.

Mingers, J. (2009). Measuring the research contribution of management academics using the Hirsch-index. Journal of the Operational Research Society, doi:10.1057/jors.2008.94

Moed, H. F. (2005). Citation Analysis in Research Evaluation. Dordrecht: Springer. ISBN 1402037139. 
Orbay, M., Karamustafaoglu, O., \& Oner, F. (2007). What does Hirsch index evolution explain us?: a case study. Turkish Journal of Chemistry, 27(8), 1-5. Rousseau, R. (2006) New developments related to the Hirsch index, E-prints in Library and Information Science (ELIS), eprints.rclis.org.

Saad, G. (2006). Exploring the $h$-index at the author and journal levels using bibliometric data of productive consumer scholars and business-related journals respectively. Scientometrics, 69(1), 117-120.

Schreiber, M. (2007). Self-citation corrections for the Hirsch index. EuroPhysics Letters, 78, doi:10.1209/0295-5075/78/30002.

Schreiber, M. (2008). A modification of the $h$-index: the $h_{m}$-index accounts for multi-authored manuscripts. http://arxiv.org/abs/0805.2000v1

Seglen, P. (1992). How representative is the journal impact factor? Research Evaluation, 2, 143-149.

Seglen, P. (1997). Why the impact factor of journals should not be used for evaluating research. British Medical Journal, 314, $498-502$.

Sidiropoulos, A., Katsaros, D., \& Manolopoulos, Y. (2007). Generalized Hirsch h-index for disclosing latent facts in citation networks. Scientometrics, 72(2), $253-280$.

Thomson Reuters (2009) http://www.thomsonreuters.com/products_services/scientific/Journal_Citation_Reports.

Van Raan, A. F. J. (2006). Comparison of the Hirsch-index with standard bibliometric indicators and with peer judgment for 147 chemistry research groups. Scientometrics, 67(3), 491-502.

Wendl, M. (2007). H-index: however ranked, citations need context. Nature, 449, p403.

Woeginger, G. H. (2008). An axiomatic characterization for the Hirsch-index. Mathematical Social Sciences, 56, $224-232$. 